Boletín de la Sociedad Geológica Mexicana

VOLUMEN 65, NÚM. 3, 2013, P. 591-607

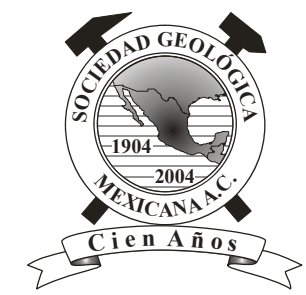

\title{
Los gonfoterios (Proboscidea, Gomphotheriidae) de Colima, México
}

\author{
Gloria Tapia-Ramírez ${ }^{1, *}$, Ana Fabiola Guzmán ${ }^{2,1}$, Oscar J. Polaco ${ }^{\dagger, 2}$ \\ ${ }^{1}$ Escuela Nacional de Ciencias Biológicas, Instituto Politécnico Nacional, Prolongación de Carpio y Plan de Ayala s/n, Santo Tomás, \\ México 11340, D.F. \\ ${ }^{2}$ Instituto Nacional de Antropología e Historia, Laboratorio de Arqueozoología "M. en C. Tícul Álvarez Solórzano", Subdirección de \\ Laboratorios y Apoyo Académico, Moneda 16, Centro Histórico, México 06060, D.F. \\ *tapiaramangel@hotmail.com
}

\begin{abstract}
Resumen
Se describen los restos de Cuvieronius hyodon, recuperados en el estado de Colima, enfatizando las características que permiten diferenciar los restos postcraneales de este gonfoterio de los de otros proboscidios mexicanos. El estudio del esqueleto permitió identificar cuatro individuos adultos, dos hembras y dos machos, e interpretar las diferencias en talla como consecuencia del dimorfismo sexual secundario. El análisis bivariante comparativo de los terceros molares inferiores ubicó la talla de la población de Colima como intermedia entre las poblaciones mexicanas (167-215 mm de longitud del molar), aunque es pequeña comparada con las poblaciones sudamericanas (197-243 mm). La distribución de las localidades de Cuvieronius en el país sugiere que la dispersión del grupo se efectuó a través de las tierras bajas de ambas vertientes, de manera similar a lo que se observa en especies actuales de afinidad tropical.
\end{abstract}

Palabras clave: Cuvieronius, Gomphotheriidae, dimorfismo, osteología, Pleistoceno.

\begin{abstract}
This study describes the remains of Cuvieronius hyodon recovered in the Mexican State of Colima, with an emphasis in the anatomical features that distinguish the postcranial remains of this gomphothere from other Mexican proboscideans. The study of the skeleton materials permitted the identification of four adult individuals, two females and two males, and the interpretation the size differences as a secondary sexual dimorphism trait. The comparative bivariate analysis of lower third molars placed the Colima population as medium-sized (167-215 mm, molar length) compared to other Mexican remains, although it is small-sized considering the South American populations (197-243 mm). The distribution of Cuvieronius Mexican localities through the country suggests that the dispersion of this gomphothere was made through the lowlands of both oceanic slopes, similar to what is observed in extant species of tropical affinity.
\end{abstract}

Keywords: Cuvieronius, Gomphotheriidae, dimorphism, osteology, Pleistocene. 


\section{Introducción}

En México, los hallazgos de mamíferos pleistocénicos son relativamente comunes, pues los sedimentos de esa edad están ampliamente distribuidos por todo el país y son muy superficiales, lo que favorece su descubrimiento, que además en muchos casos es fortuito. Algunos estados (e.g. Puebla, Distrito Federal y Estado de México) tienen un registro fósil más amplio que otros, donde el registro fósil es más bien escaso o nulo (e.g. Colima, Durango y Quintana Roo) (Arroyo-Cabrales et al., 2002, 2010; FerrusquíaVillafranca et al., 2010).

Uno de los grupos de mamíferos mejor representado en el registro fósil mexicano es el orden Proboscidea, con presencia en el Mioceno tardío (Gomphotherium), el Plioceno (Rhynchotherium y Stegomastodon) y el Pleistoceno (Cuvieronius, Stegomastodon [?], Mammut y Mammuthus) (Polaco, 2002).

De las tres familias de proboscidios registradas en el país, la Elephantidae está constituida por dos especies del género Mammuthus; este taxón está ampliamente distribuido a lo largo del territorio nacional (Arroyo-Cabrales et al., 2007, 2010). La familia Mammutidae está representada por una sola especie (Mammut americanum) y ha sido registrada en siete estados del país (Arroyo-Cabrales et al., 2010). La familia Gomphotheriidae, la más diversa, está representada por cuatro géneros y quizá cinco especies: Gomphotherium, Rhynchotherium y Stegomastodon son géneros poco comunes y se conocen de seis, once y cinco localidades, respectivamente (Alberdi y Corona-M., 2005; Arroyo-Cabrales et al., 2007), mientras que Cuvieronius es el mejor representado, con restos en 44 localidades en 17 estados, principalmente en el centro y sur del país (Figura 1, Tabla 1).

Cuvieronius fue el género más tardío de los gonfoterios, sobrevivió hasta finales del Pleistoceno (Lambert, 1996). El número de especies válidas en México es todavía tema de discusión y se han propuesto de una a cuatro especies nominales, tres de ellas basadas en material mexicano [ $C$. tropicus (Cope, 1884), C. oligobunis (Cope, 1893), C. arellanoi (Ochoterena y Silva-Bárcenas, 1970)] y la cuarta descrita con material sudamericano [C. hyodon (Fischer, 1814)].

Excepto Cuvieronius arellanoi, que es omitida por los especialistas del grupo (e.g. Laurito, 1988; MontellanoBallesteros, 2002), las especies fueron descritas en función

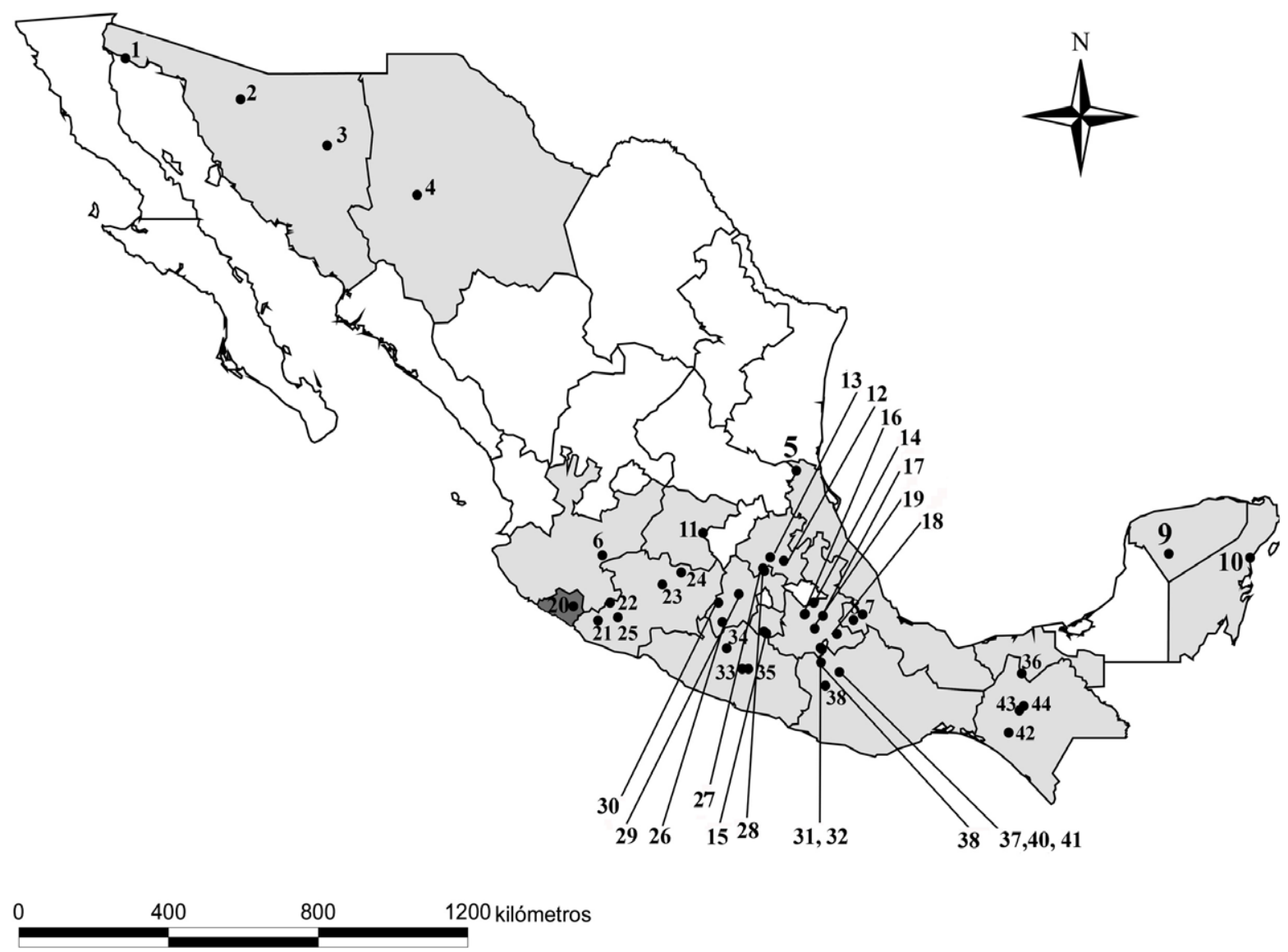

Figura 1. Localidades de gonfoterios citados como Cuvieronius en el país. Los números se refieren a los usados en la Tabla 1. 
Tabla 1. Localidades con hallazgos de gonfoterios citados como Cuvieronius en la República Mexicana.

\begin{tabular}{|c|c|}
\hline Localidad, Estado & Cita \\
\hline 1 El Golfo de Santa Clara, Sonora & Shaw, 1981 \\
\hline 2 Oquitoa, Sonora & Lucas y González-León, 1997 \\
\hline 3 Térapa, Sonora & Mead et al ., 2006 \\
\hline 4 Ciudad Guerrero, Chihuahua & Eaton, 1905 \\
\hline 5 Ébano, San Luis Potosí & Polaco y Aguilar, 1999 \\
\hline 6 Lago de Chapala, Jalisco & Downs, 1958; Lucas 2003 \\
\hline 7 Atoyac, Veracruz & Peña Serrano et al ., 2004 \\
\hline 8 Rincón de Maravillas, Veracruz & Peña Serrano et al ., 2004; Peña Serrano y Miranda Flores, 2009 \\
\hline 9 Cueva de Loltún, Yucatán & Álvarez, 1982 \\
\hline 10 Nai Tucha, Quintana Roo & Polaco et al ., 2002 \\
\hline 11 La Purísima, Guanajuato & Polaco, 2008 \\
\hline 12 Real del Monte, Hidalgo & Freudenberg, 1922 \\
\hline 13 Santa Cruz Amajac, Hidalgo & Castillo et al ., 1996 \\
\hline 14 Acultzingo, Puebla & Alberdi y Corona-M., 2005 \\
\hline 15 Rancho Gerardo, Puebla & Montellano-Ballesteros, 2002 \\
\hline 16 San Baltazar Tetela, Puebla & Pichardo del Barrio, 1960 \\
\hline 17 Tecamachalco, Puebla & Felix y Lenk, 1891 \\
\hline 18 Tehuacán, Puebla & Pichardo del Barrio, 1960 \\
\hline 19 Valsequillo, Puebla & Miller y Carranza-Castañeda, 1984 \\
\hline 20 Los Ortices, Colima & Este trabajo \\
\hline 21 Hihuitlán, Michoacán & Guzmán, obs. pers. 2004 \\
\hline 22 Tepalcatepec, Michoacán & Guzmán com. pers. \\
\hline 23 Tzintzuntzan, Michoacán & Robles-Camacho et al ., 2010 \\
\hline 24 Cuto del Porvenir, Michoacán & García-Zepeda, 2006 \\
\hline 25 Cerro del Pino, Michoacán & Polaco, 1988 \\
\hline 26 Almoloya, México & Del Castillo, 1869 \\
\hline 27 Apaxco de Ocampo, México & Cope, 1884 \\
\hline 28 Tequixquiac, México & Cope, 1884 \\
\hline 29 Quetepec, México & Freudenberg, 1922 \\
\hline 30 Ixtapatongo, México & Pichardo del Barrio, 1960 \\
\hline 31 Cueva Encantada de, Chimalacatlán, Morelos & Pichardo del Barrio, 1960 \\
\hline 32 Nexpa, Morelos & Corona-M. y Alberdi, 2006 \\
\hline 33 Chichihualco, Guerrero & Freudenberg, 1922 \\
\hline 34 Nanacatla, Guerrero & Torres-Martínez, 1981 \\
\hline 35 Zumpango del río, Guerrero & Alberdi y Corona-M., 2005 \\
\hline 36 Teapa, Tabasco & Polaco, 2005 (com. pers.) \\
\hline 37 Cosoltepec, Oaxaca & Ochoterena y Silva-Bárcenas, 1970 \\
\hline 38 Huajuapan de León, Oaxaca & Felix y Lenk, 1891 \\
\hline 39 El Pedernal, Oaxaca & Jiménez-Hidalgo et al ., 2011 \\
\hline 40 Santa Catalina Chinango, Oaxaca & Ochoterena y Silva-Bárcenas, 1970 \\
\hline 41 Tlaxiaco, Oaxaca & Felix y Lenk, 1891 \\
\hline 42 Villa Corzo, Chiapas & Böese, 1905 \\
\hline 43 Chiapa de Corzo, Chiapas & Carbot-Chanona et al ., 2008 \\
\hline 44 Ixtapa, Chiapas & Ferrusquía-Villafranca et al ., 2010 \\
\hline
\end{tabular}


de la morfología de los molares inferiores terceros $\left(\mathrm{M}_{3}\right)$. Estos dientes son bunodontos y están constituidos por cúspides generalmente denominadas cónidos; los cónidos están arreglados transversalmente en pares llamados lófidos, crestas o colinas, terminando en un talónido; además, entre los cónidos del lado labial (también conocidos como pretritos en los molares inferiores) existen cúspides accesorias llamadas cónulos. Las especies mexicanas $C$. oligobunis y $C$. tropicus están basadas en variaciones del número de los lófidos (4 a 4 1/4 vs. 5); sin embargo, se ha observado que el último lófido del $\mathrm{M}_{3}$ varía en tamaño y forma, lo que dificulta discernir en muchos casos si se trata realmente de un lófido más o de un talón muy grande y resulta ser un carácter muy variable para separar estas especies (e.g. obs. pers.; Simpson y Paula Couto, 1957; Laurito, 1988; Montellano-Ballestero, 2002). La variación de la estructura del $\mathrm{M}_{3}$ tampoco permite separar las poblaciones mexicanas de las centro y sudamericanas, $\mathrm{y}$ así algunos autores consideran que se trata de una sola especie (Polaco, 2002). Esa especie tendría una amplia distribución continental (desde el sureste de Estados Unidos hasta Argentina) y debiese nombrarse, por principio de prioridad, como Cuvieronius hyodon, criterio al cual se apega este trabajo.

Los restos de Cuvieronius que normalmente se recuperan en México son dientes aislados y eventualmente asociados a partes mandibulares y craneales, el registro en Puebla de Montellano-Ballesteros (2002) es el único que adicionalmente reporta y describe algunos elementos postcraneales. Villada (1897) menciona ocho piezas postcraneales asociados a una mandíbula completa, ésta última designada por Cope (1893) como tipo de la ahora especie Cuvieronius oligobunis; las investigaciones posteriores sólo mencionan a la mandíbula, la cual está en resguardo del Instituto de Geología de la UNAM.

En esta ocasión se presenta información sobre los materiales de Cuvieronius encontrados en Colima, la mayoría de ellos elementos postcraneales, recuperados junto con dientes, vértebras y otros restos pertenecientes a Eremotherium laurillardi. Este trabajo es el primer registro estatal formal de vertebrados pleistocénicos, aun cuando ya ha sido incluido en obras generales sobre la distribución de la fauna fósil (Arroyo-Cabrales et al., 2007, 2010).

Dado que las piezas postcraneales de Cuvieronius son escasas en el registro fósil, se consideró conveniente aportar elementos para discriminarlas respecto a las de otros proboscidios registrados en México, así como proporcionar datos morfométricos y del dimorfismo sexual y comentar aspectos de la distribución de este género en el país.

\section{2. Área de estudio}

Los restos fueron recuperados en 1988 por los arqueólogos María de los Ángeles Olay y Pedro Francisco Sánchez Nava, en el cerro La Guayabilla (19²'36.93"N, $\left.103^{\circ} 42^{\prime} 58.03^{\prime \prime O}, 374 \mathrm{msnm}\right), 30 \mathrm{~km}$ al sur de la capital del estado de Colima y $6 \mathrm{~km}$ al sureste del ejido Los Ortices (Figura 2). La localidad está próxima al río El Salado, en la cuenca del río Coahuayana. La vegetación en los alrededores, de acuerdo a la clasificación de Rzedowski (1994), es un bosque tropical caducifolio con zonas de matorral subinerme. El clima que predomina es de tipo cálido subhúmedo con lluvias en verano $\left(\mathrm{Aw}_{\mathrm{o}}(\mathrm{w})\right)$ (García, 1973). En la localidad afloran rocas del Terciario y formaciones cretácicas (INEGI, 1981), cubiertas en áreas por sedimentos pleistocénicos y recientes.

\section{Material y métodos}

El material craneal y dental fue identificado siguiendo a Osborn $(1936,1942)$ y Laurito (1988). Para el estudio detallado del esqueleto postcraneal, se utilizó el atlas elaborado por Olsen (1979) para Mammut y Mammuthus, proboscidios que coexistieron temporalmente con Cuvieronius. También se usaron las descripciones de otros gonfoterios que habitaron en México, independientemente de su contemporaneidad o no con Cuvieronius y aun cuando estuviesen basadas en material extranjero por no conocerse restos poscraneales en nuestro país: para Stegomastodon se usó a Alberdi et al. (2009) y Lucas et al. (2011); para Rhynchotherium, la descripción de Pasenko (2007); y para Gomphotherium, a Tassy (1977) y Göhlich (1998, 2010). Adicionalmente, se consultaron ejemplares de Mammuthus de la Colección Paleontológica del Laboratorio de Arqueozoología "M. en C. Ticul Álvarez Solórzano" y las descripciones de restos sudamericanos de Cuvieronius (Boule y Thevenin, 1920). La sección comparativa es resultado de conclusiones propias, apoyadas en los rasgos ilustrados o descritos por los autores mencionados, quienes no necesariamente hacen observaciones comparativas. La terminología anatómica empleada para describir las particularidades de los restos se apegó en lo general a Olsen (1979) y Mazo-Pérez (1995).

Los huesos fueron medidos siguiendo el criterio de Von den Driesch (1976) y Ficcarelli et al. (1993). El número mínimo de individuos fue establecido con el material postcraneal, usando criterios anatómicos de talla y de edad, separando los elementos de la muestra en izquierdos y derechos y considerando luego su tamaño relativo junto con el grado de fusión epifisaria, para ser asignados a un mismo individuo o a uno diferente, como proponen Klein y Cruz-Uribe (1984).

La notación de los molares siguió a DeBlase y Martin (1974), usando la letra M para el molar; acompañada por un número que indica la posición del diente (primero, segundo, tercero) y que aplicado como subíndice señala que se trata de un diente inferior. Las medidas tomadas fueron las convencionales del grupo (longitud total y anchuras de cada lófido y del talónido) y han sido consideradas al menos desde Osborn (1923). Con ellas se calculó el índice de anchura de 


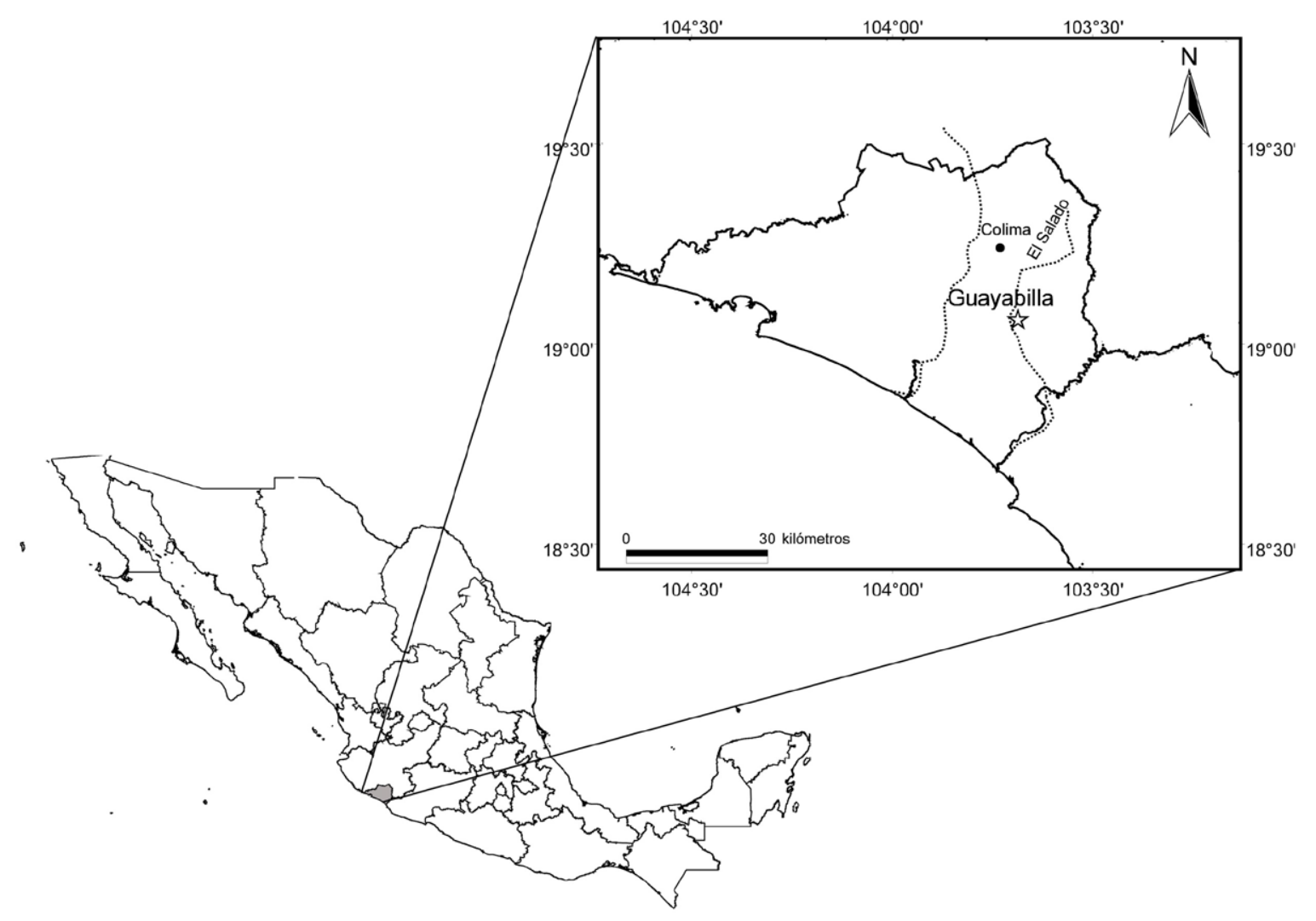

Figura 2. Ubicación del área de estudio.

los molares, valor típicamente usado para los gonfoterios (e.g. Osborn, 1923; Tassy, 1977; Göhlich, 1998). El tamaño del molar se comparó con el de otros molares recuperados en México y otros países de América (Lucas y González-León, 1997; Frassinetti y Alberdi, 2000; Montellano-Ballesteros, 2002; Alberdi et al., 2004; Alberdi y Corona-M., 2005; Chávez-Aponte et al., 2008 y Robles-Camacho et al., 2010), mediante un análisis bivariante entre la longitud total y la anchura máxima (Göhlich, 1998; Frassinetti y Alberdi, 2000), a través del programa SigmaPlot versión 10.0 (Systat Software, 2006).

El material estudiado quedó alojado en la colección paleontológica del Laboratorio de Arqueozoología "M en C Ticul Álvarez Solórzano" de la Subdirección de Laboratorios y Apoyo Académico del INAH, cuyo acrónimo es DP.

\section{Sistemática Paleontológica}

Familia Gomphotheriidae Cabrera, 1929 Género Cuvieronius Osborn, 1923

\section{Cuvieronius hyodon (Fischer, 1814)} Tablas 2 y 3, Figuras 3-9

Diagnosis: Especie brevirrostrina (presencia de una sínfisis mandibular fuertemente abreviada) que no posee defensas inferiores. Las defensas superiores tienen una banda de esmalte dispuesta en forma espiral. Los dientes mandibulares son bunodontos y al desgastarse forman figuras de tréboles simples de contorno simple a complejo. El tercer molar tiene entre cuatro a cinco lófidos (Osborn, 1936, 1942).

\subsection{Material examinado}

$\mathrm{Un}_{3}$ derecho aislado (DP-5789); una rama mandibular derecha con $\mathrm{M}_{3}$ in situ (DP-5790); un fragmento distal de defensa (DP-5791); un fragmento proximal de defensa (DP-5792); dos espinas neurales (DP-5793 y DP-5794); tres fragmentos de costillas (DP-5815, DP-5815A y DP5819); un húmero derecho (DP-5795); una ulna izquierda (DP-5796); dos huesos innominados derechos (DP-5797 y DP-5798), uno izquierdo (DP-5799); dos fémures izquierdos (DP-5800 y DP-5801), dos fémures derechos (DP-5803 y DP-5804); una diáfisis de fémur derecho (DP-5802); dos tibias derechas (DP-5805 y DP-5806); dos tibias izquierdas (DP-5807 y DP-5808); una fíbula izquierda (DP-5809) y una porción distal de fíbula (DP-5816); un astrágalo izquierdo (DP-5810); dos calcáneos derechos, uno completo (DP-5811) y el otro roto (DP-5812); un calcáneo izquierdo (DP-5813). 


\subsection{Descripción del material}

\subsubsection{Elementos dentales}

Se rescataron un molar aislado, una rama mandibular $\mathrm{y}$ dos fragmentos de defensas. El molar es un $\mathrm{M}_{3}$ derecho (DP-5789) bunodonto, con cinco lófidos (el último de los cuales es angosto y carece de cónulos accesorios) y un pequeño talónido (semejante a una protuberancia); los cónidos labiales (también conocidos como pretritos en los molares inferiores) están ligeramente retrasados respecto a los linguales (postritos), la figura oclusal formada es la de un trébol simple, de contorno simple (Figura 3a). La pieza es más o menos rectangular y tiene la corona muy desgastada, conservando apenas $1 \mathrm{~cm}$ de ella. En los dos lófidos más anteriores, la figura oclusal de ambos cónidos se ha fusionado y en el último lófido el desgaste es evidente pero no tan avanzado, ya que la figura de cada cónido está perfectamente separada. El molar conserva, entre las raíces, un fragmento de rama horizontal de la mandíbula (Figura 3b). La longitud total del molar es $183 \mathrm{~mm}$ y las anchuras son las siguientes: $1^{\circ}$ lófido, $74.0 \mathrm{~mm} ; 2^{\circ}$ lófido, $75.0 \mathrm{~mm}$; $3^{\circ}$ lófido, $72.0 \mathrm{~mm}$; $4^{\circ}$ lófido, $63.0 \mathrm{~mm}$; $5^{\circ}$ lófido, 42.0 mm; talónido, $12.5 \mathrm{~mm}$; el índice de anchura es de $40.9 \%$.

La rama mandibular (DP-5790) está incompleta pero conserva la rama horizontal, parte de la rama ascendente y la sínfisis mandibular brevirrostrina (Figura 3c). La pieza es robusta, convexa del lado labial y carece de evidencias de haber portado defensas en alguna etapa de su vida. Mide $616 \mathrm{~mm}$ de longitud, $150 \mathrm{~mm}$ de altura al nivel anterior de la serie molar y $149 \mathrm{~mm}$ al nivel posterior de la serie molar.
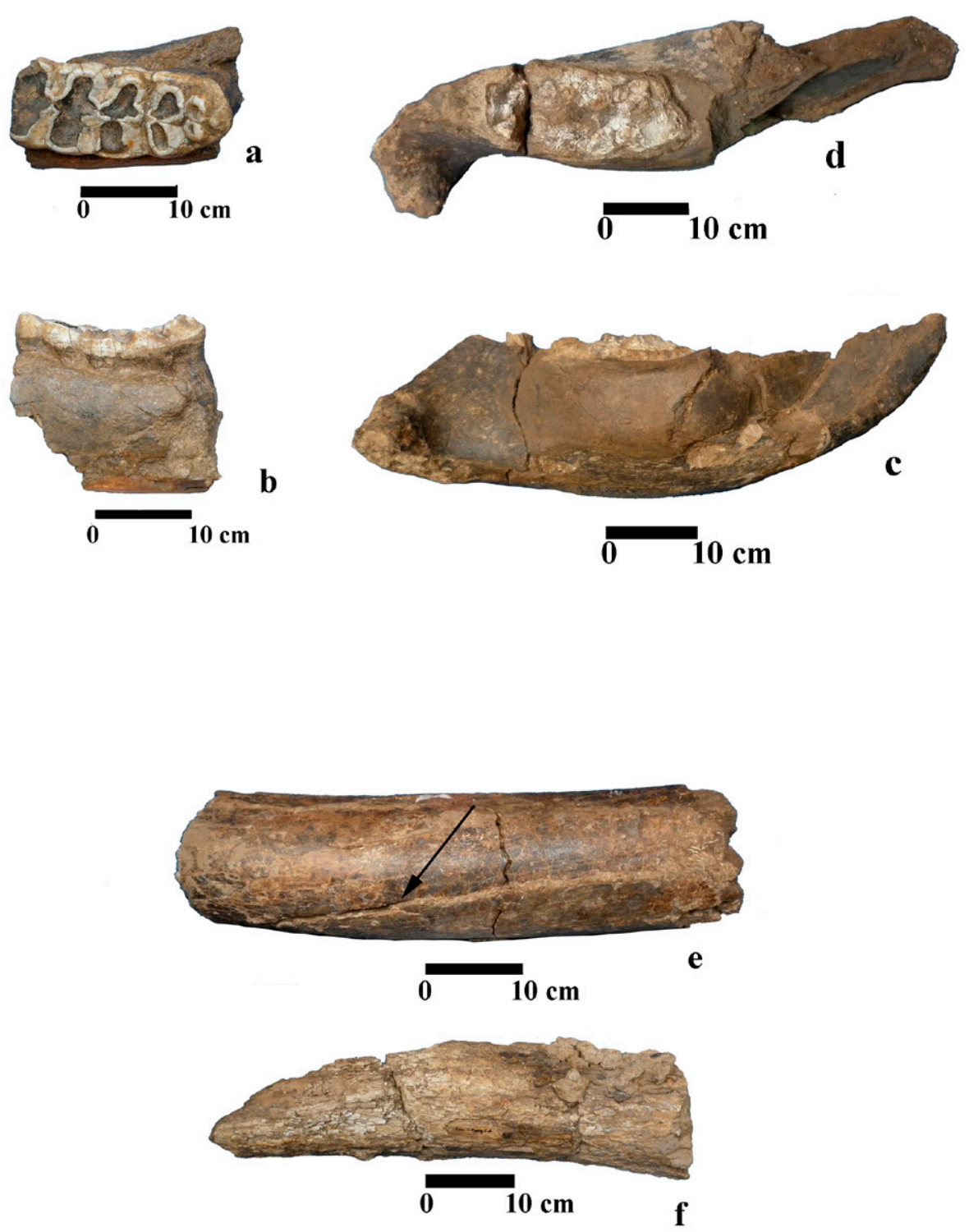

Figura 3. Elementos dentales. a) M3 derecho (DP-5789), vista oclusal, b) misma pieza, vista lingual; c) rama mandibular derecha (DP-5790), vista lingual, d) misma pieza, vista oclusal; e) fragmento proximal de defensa (DP-5792), f) fragmento distal de defensa (DP-5791). La flecha indica la huella dejada por la banda de esmalte en la defensa. 
Conserva el $\mathrm{M}_{3}$ dañado en casi toda su superficie (Figura $3 d$ ), y tiene alrededor de $207.0 \mathrm{~mm}$ de longitud, $93.5 \mathrm{~mm}$ de anchura máxima y un índice de $45.1 \%$. La rama mandibular y el diente están fisurados a la misma altura.

De los fragmentos de defensa, uno corresponde al extremo proximal (DP-5792): está roto en dos porciones, es de forma oval en sección transversal, mide $490 \mathrm{~mm}$ de longitud y $435 \mathrm{~mm}$ de perímetro y conserva restos de la banda de esmalte y de la marca dejada por ésta, la cual tiene $40.0 \mathrm{~mm}$ de anchura aproximada; la torsión de la defensa y de la banda de esmalte es muy evidente (Figura 3e). El otro fragmento es distal (DP-5791; Figura 3f) y corresponde al ápice de una defensa: está roto en tres porciones y se va angostando distalmente, terminando en una punta aplanada; no conserva evidencias de esmalte, dado el estado tan deteriorado que presenta, pero se observa cierta curvatura, propia del género Cuvieronius.

\subsubsection{Esqueleto axial}

Se recuperaron partes de dos vértebras y tres costillas. En el primer caso, se trata de dos espinas neurales de vértebras torácicas. Una presenta restos del arco (DP-5794; Figura 4a) y tiene una longitud máxima de $300 \mathrm{~mm}$ y una anchura entre las postzigoapófisis de $105 \mathrm{~mm}$. La otra espina no conserva el arco (DP-5793; Figura 4b), tiene una longitud de $260 \mathrm{~mm}$ y una anchura de $95 \mathrm{~mm}$. El cuerpo de la espina es triangular, esto es, más ancho en la base y adelgazado hacia el extremo libre, en donde se ensancha en un botón óseo apical. En la cara posterior presenta un surco, más profundo en la base y somero hacia la punta.

Respecto a las costillas, una de ellas (DP-5819; Figura 4c) es una porción proximal, muy curvada y comprimida, que conserva restos de la cabeza articular, de forma roma, y del tubérculo, el cual es pequeño y ovalado; tiene una
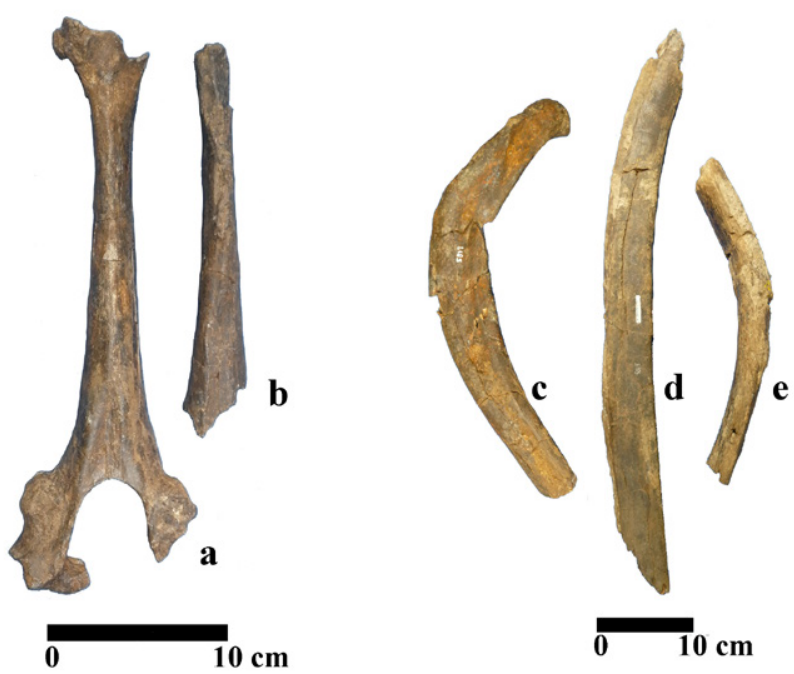

Figura 4. Esqueleto axial. a) Espina neural con restos del arco (DP-5794); b) espina neural (DP-5793); c) costilla (DP-5819); d) costilla (DP-5015); e) costilla (DP-5815-A). longitud de $410 \mathrm{~mm}$. Otra costilla (DP-5815; Figura 4d) corresponde a la porción media del cuerpo, punto en el cual es gruesa y poco curva, con un notorio surco costal que corre a todo su largo, esta pieza mide $540 \mathrm{~mm}$. La tercera pieza (DP-5815A; Figura 4e) corresponde a una porción intermedia entre las dos anteriores, ya que es gruesa pero también curva y se alcanza a notar el surco costal; tiene una longitud de $310 \mathrm{~mm}$.

\subsubsection{Esqueleto apendicular}

Del miembro anterior se recuperaron un húmero y una ulna. El húmero (DP-5795; Figuras 5a y 5b) es robusto y bajo, está casi completo y tiene todas las suturas fusionadas. En la epífisis proximal, la cabeza articular es relativamente ancha y la tuberosidad lateral, aunque rota, es un poco más alta que la cabeza articular. En la diáfisis se observa un moderado desarrollo de la cresta deltoidea, el surco musculoespiral más o menos profundo y bien
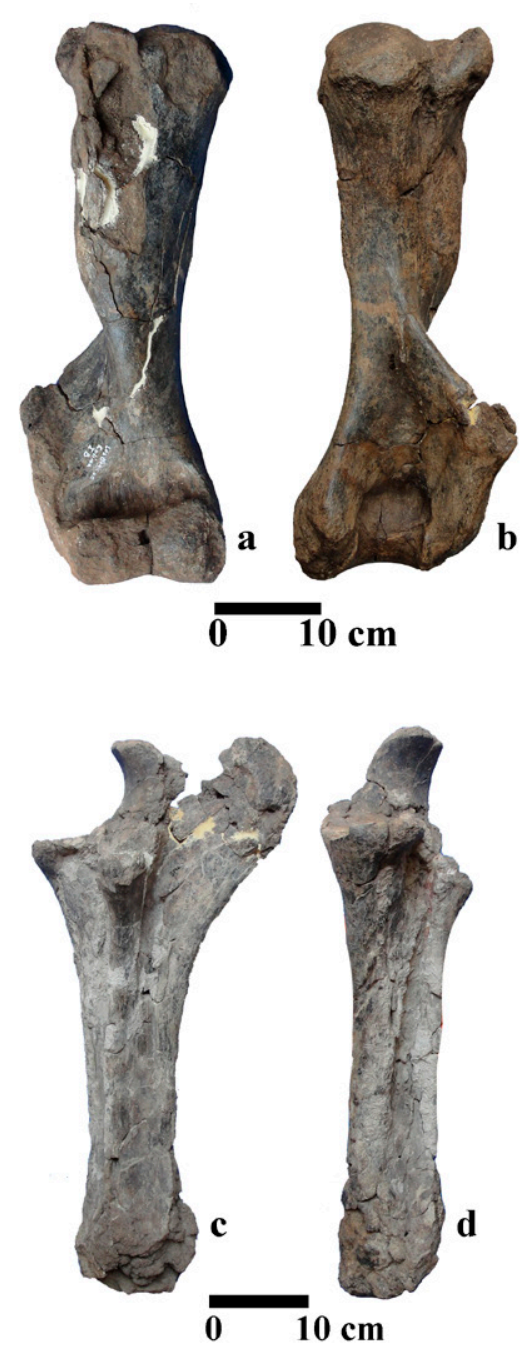

Figura 5. Esqueleto apendicular, miembro anterior. a) Húmero derecho (DP-5795), vista anterior; b) misma pieza, vista posterior; c) ulna izquierda (DP-5796), vista lateral; d) misma pieza, vista anterior. 
definido y la cresta supracondiloidea lateral notablemente desarrollada. En la epífisis distal, el epicóndilo medial es ligeramente más grande y proyectante que el lateral, y este último no se percibe por ser continúo con la cresta supracondiloidea. Las dimensiones del húmero son: longitud máxima, $560 \mathrm{~mm}$; anchura de la epífisis distal, $184 \mathrm{~mm}$; anchura de la epífisis proximal, $174 \mathrm{~mm}$; anchura más pequeña de la diáfisis, $84 \mathrm{~mm}$; extensión máxima de la cresta supracondiloidea, $182.2 \mathrm{~mm}$; distancia del punto más saliente de la cresta supracondiloidea lateral a la tuberosidad lateral, aproximadamente $204.0 \mathrm{~mm}$; distancia del punto más saliente de la cresta supracondiloidea lateral al cóndilo lateral, $248.5 \mathrm{~mm}$; diámetro transversal articular de la epífisis distal, $160.6 \mathrm{~mm}$; diámetro de la diáfisis a nivel de la extensión máxima de la cresta deltoidea, $178.2 \mathrm{~mm}$.

La ulna (DP-5796; Figuras 5c y 5d) es una pieza alargada, con la diáfisis irregularmente prismática. En la epífisis proximal, el proceso olecranon está incompleto y el proceso anconeano es muy ancho. El extremo distal está roto. El hueso tiene las siguientes dimensiones: $725.0 \mathrm{~mm}$ de longitud, $138.1 \mathrm{~mm}$ de anchura distal de la diáfisis, 120.1 mm de anchura mínima de la diáfisis y la anchura mayor del proceso anconeano es de $82.0 \mathrm{~mm}$.

Con respecto a la extremidad posterior, se recuperaron tres huesos innominados o coxales, cinco fémures y cuatro tibias, una fíbula y varios elementos del pie.

El hueso innominado más completo es derecho (DP5798; Figura 6a), mide $950 \mathrm{~mm}$ de longitud y conserva casi todo el ilion, el cual tiene forma de abanico; el pubis está roto en su porción anterior y el isquion está casi completo, pero le falta la tuberosidad; el isquion y el pubis son delgados y bordean un amplio foramen obturador, el cual está incompleto; el acetábulo es poco profundo, con las suturas articulares obsoletas y $170 \mathrm{~mm}$ de diámetro anteroposterior. Un segundo hueso innominado derecho (DP-5797) es de menores proporciones; tiene una longitud conservada de $450 \mathrm{~mm}$ y sólo presenta partes del ilion y del isquion, así como el acetábulo completo, cuyo diámetro anteroposterior es de $140 \mathrm{~mm}$. El tercer hueso innominado (DP-5799) es izquierdo, está muy deteriorado y conserva una porción del ilion y del acetábulo; tiene una longitud conservada de $750 \mathrm{~mm}$.

Los fémures son notablemente esbeltos, sobre todo en la parte media de la diáfisis (Figuras 6 b y $6 c$ ). La superficie de la cara anterior es aplanada en la mitad proximal, mientras que la mitad distal es elevada (Figura 6b). En la epífisis proximal, el trocánter mayor está expandido, la fosa trocantérica es somera y la cabeza articular está dirigida laterad (Figura 6c); el cuello de la cabeza articular es corto y describe, junto con la diáfisis, una curva poco pronunciada. En la epífisis distal, el cóndilo medial es más grande que el lateral. Cuatro fémures presentan fusión epifisaria (DP5800, DP-5801, DP-5803 y DP-5804); el quinto fémur (DP-5802) está roto en ambos extremos y sólo conserva la diáfisis. Sus dimensiones se proporcionan en la Tabla 2.

Las tibias están más o menos conservadas (DP-5805,
DP-5806, DP-5808), aunque la pieza más grande es la más dañada (DP-5807). En este hueso (Figuras 6d y 6e), la diáfisis es prismática de cuatro caras; la cresta tibial está bien desarrollada y corre a todo lo largo de la diáfisis en su cara anterior; la cara posterior es aplanada. La epífisis proximal en todos los casos está rota en su cara anterior, pero permite observar que los cóndilos medial y lateral están bien definidos (el lateral más bajo que el medial) y que la espina tibial no es prominente. El maléolo medial no es exageradamente prominente. En la Tabla 2 se proporcionan las dimensiones de estas piezas.

La fíbula (DP-5809; Figuras 6f-6h) es muy delgada, con la diáfisis más ancha en su parte distal y muy aplanada en la parte media, aparentando sufrir torsión en ese punto; el borde externo de la diáfisis es recto, mientras que el borde interno es convexo en la parte media y distal. La epífisis proximal o cabeza es ancha y plana y posee una carilla articular interna en el punto de contacto con la tibia. La epífisis distal es más amplia que la epífisis proximal, presentando una cavidad interna para articular con el extremo distal lateral de la tibia (Figura 6g). La longitud máxima es de $536 \mathrm{~mm}$.

Del pie se recuperaron un astrágalo y tres calcáneos. El astrágalo (DP-5810; Figuras 7a-7c) es un hueso deprimido e irregular, de contornos redondeados, la cara medial es más pequeña que la lateral. La tróclea, en la cara dorsal, es corta y redonda y en vista posterior desciende bruscamente. Articula con el calcáneo mediante dos carillas ventrales, una de ellas de forma oval y la otra crescéntica. Mide $122 \mathrm{~mm}$ de longitud mediolateral, $99 \mathrm{~mm}$ de longitud anteroposterior y $66.6 \mathrm{~mm}$ de altura.

De los calcáneos derechos, sólo uno está completo (DP5811; Figuras 7d y 7e) y permite apreciar que la tuberosidad es baja, ligeramente redondeada y con un cuello bajo, mientras que el sustentáculo es redondeado y corto, carece de procesos y está dirigido laterad. La cara anterior presenta dos superficies articulares con el astrágalo, de forma elíptica; y mide $169 \mathrm{~mm}$ de longitud total. El otro calcáneo (DP5812) tiene la cara anterior rota y por ello las carillas de articulación no están unidas a la pieza; la longitud mayor conservada es de $154 \mathrm{~mm}$. El tercer calcáneo es izquierdo (DP-5813), está casi completo y mide $166.2 \mathrm{~mm}$ de longitud.

4.3. Número de individuos, dimorfismo sexual y talla en los gonfoterios de Colima.

La fusión completa de las piezas postcraneales, la presencia de $\mathrm{M}_{3}$ funcionales y el grado de desgaste dental, indican que todos los restos pertenecen a individuos adultos. La talla relativa de los fémures y las tibias y su lateralidad permitieron reconocer cuatro individuos: los individuos 1 y 2 son de talla grande, el individuo 3 es mediano y el individuo 4 es el más pequeño (Tabla 2; Figura 8). El individuo 1 está representado por los dos fémures y la tibia más grandes (Figura 8a); del individuo 2 se conserva únicamente la diáfisis de un fémur derecho (Figura 8b); el 

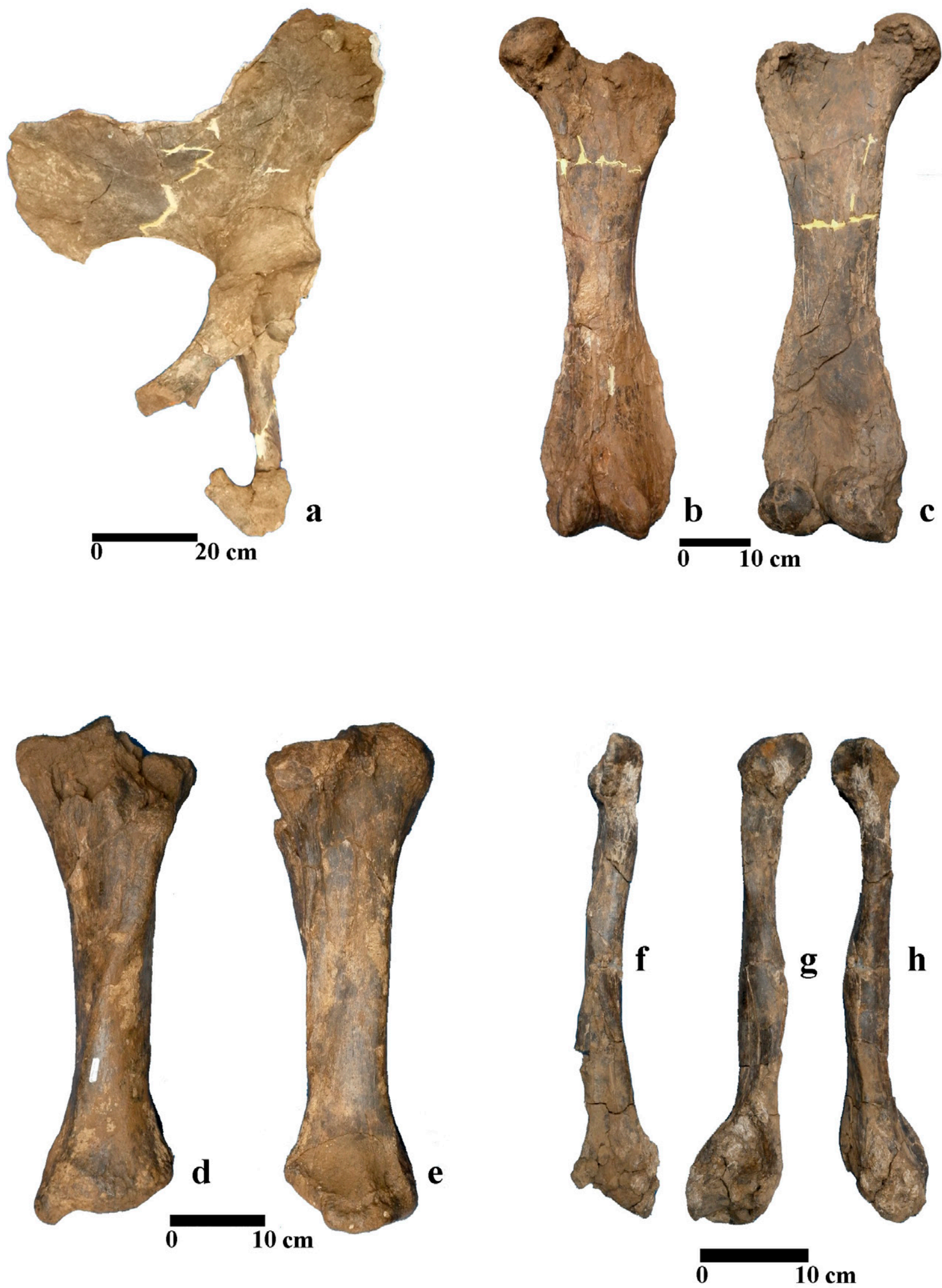

Figura 6. Esqueleto apendicular, miembro posterior. e) Hueso innominado derecho (DP-5798), vista posterior; f) fémur izquierdo (DP-5804), vista anterior; g) misma pieza, vista posterior; a) tibia derecha (DP-5806), vista anterior; b) misma pieza, vista posterior; c) fíbula izquierda (DP-5809), vista anterior, d) misma pieza, vista medial, e) misma pieza, vista lateral. 


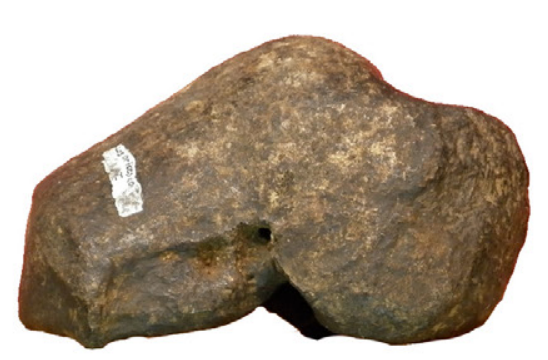

$\mathbf{a}$
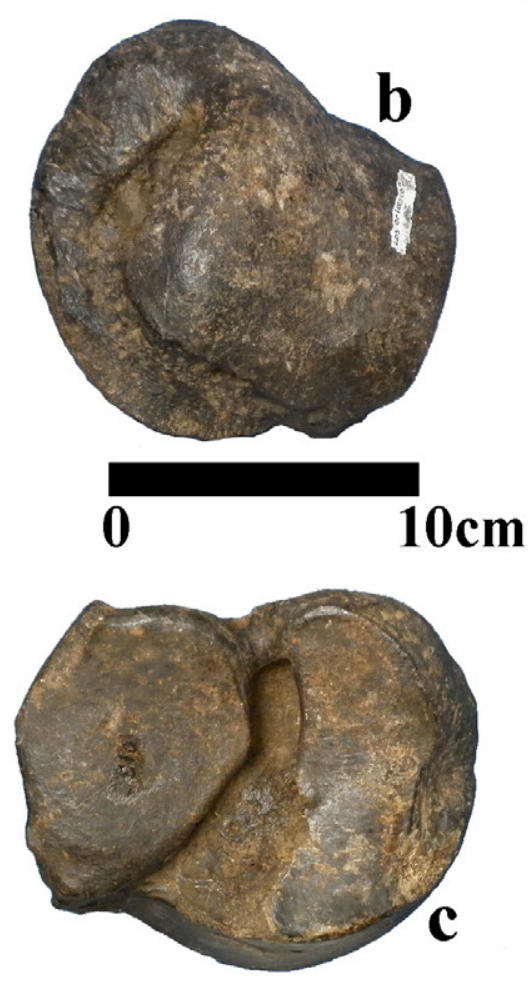

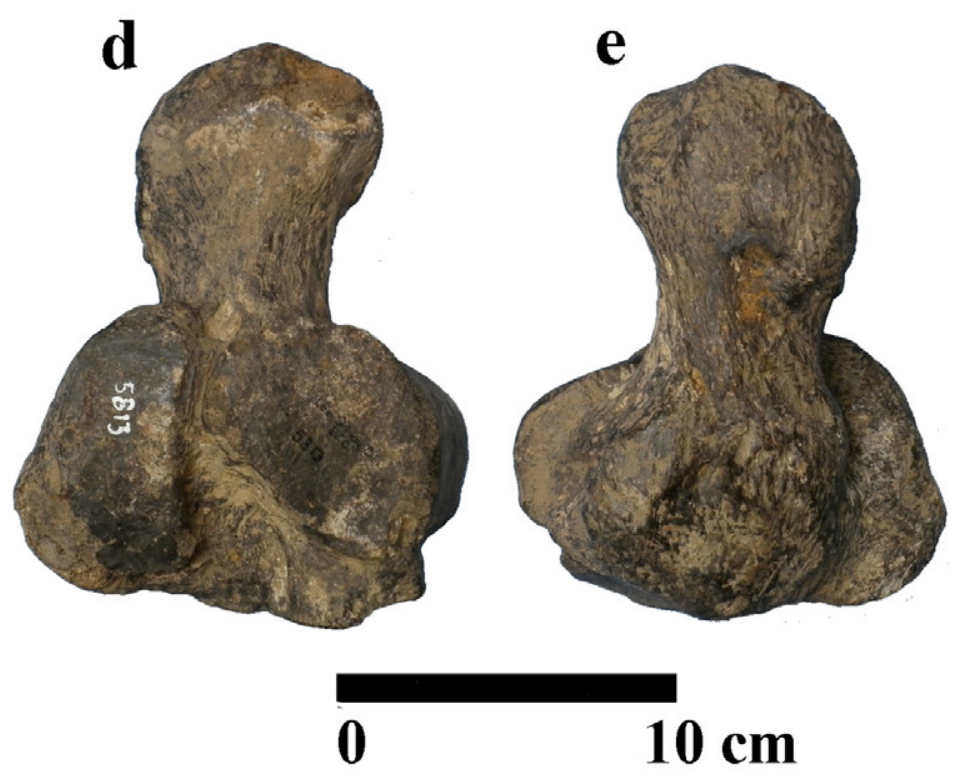

Figura 7. Elementos del pie. a) Astrágalo izquierdo (DP-5810), vista posterior, b) misma pieza, vista dorsal, c) misma pieza, vista ventral; d) calcáneo derecho (DP-5811), vista anterior o dorsal; e) misma pieza, vista posterior o ventral.

Tabla 2. Dimensiones de los fémures y tibias (clasificación de los datos por individuo).

\begin{tabular}{|c|c|c|c|c|c|c|}
\hline Individuo & Pieza & $\mathbf{L x}$ & Ap & Ad & Am & Sexo \\
\hline \multirow{3}{*}{1} & Fémur derecho (DP-5804) & 902 & 259.05 & 205.4 & 125.3 & \multirow{3}{*}{$\hat{\sigma}$} \\
\hline & Fémur izquierdo (DP-5800) & 898 & 337.1 & 212.7 & 137.3 & \\
\hline & Tibia izquierda (DP-5807) & 578 & 196 & 178 & 93.6 & \\
\hline 2 & Fémur derecho (DP-5802) & -579 & --- & --- & 111.35 & $0^{\lambda}$ \\
\hline \multirow{4}{*}{3} & Fémur izquierdo (DP-5801) & 730 & --- & 180.9 & 102.4 & \multirow{4}{*}{ of } \\
\hline & Fémur derecho (DP-5803) & 730 & -266.1 & 162.54 & 96.65 & \\
\hline & Tibia izquierda (DP-5808) & 516 & 197.2 & 150.1 & 86 & \\
\hline & Tibia derecha (DP-5806) & 515 & -199.8 & 150.6 & 87 & \\
\hline 4 & Tibia derecha (DP-5805) & 457 & -154.1 & 122 & 71 & q \\
\hline
\end{tabular}

Lx: longitud máxima, Ap: anchura de la epífisis proximal, Ad: anchura de la epífisis distal, Am: anchura más pequeña de la diáfisis. Las medidas están dadas en milímmetros. Entre paréntesis se colocaron las medidas incompletas de las piezas rotas. Los guiones indican la ausencia de dato. 

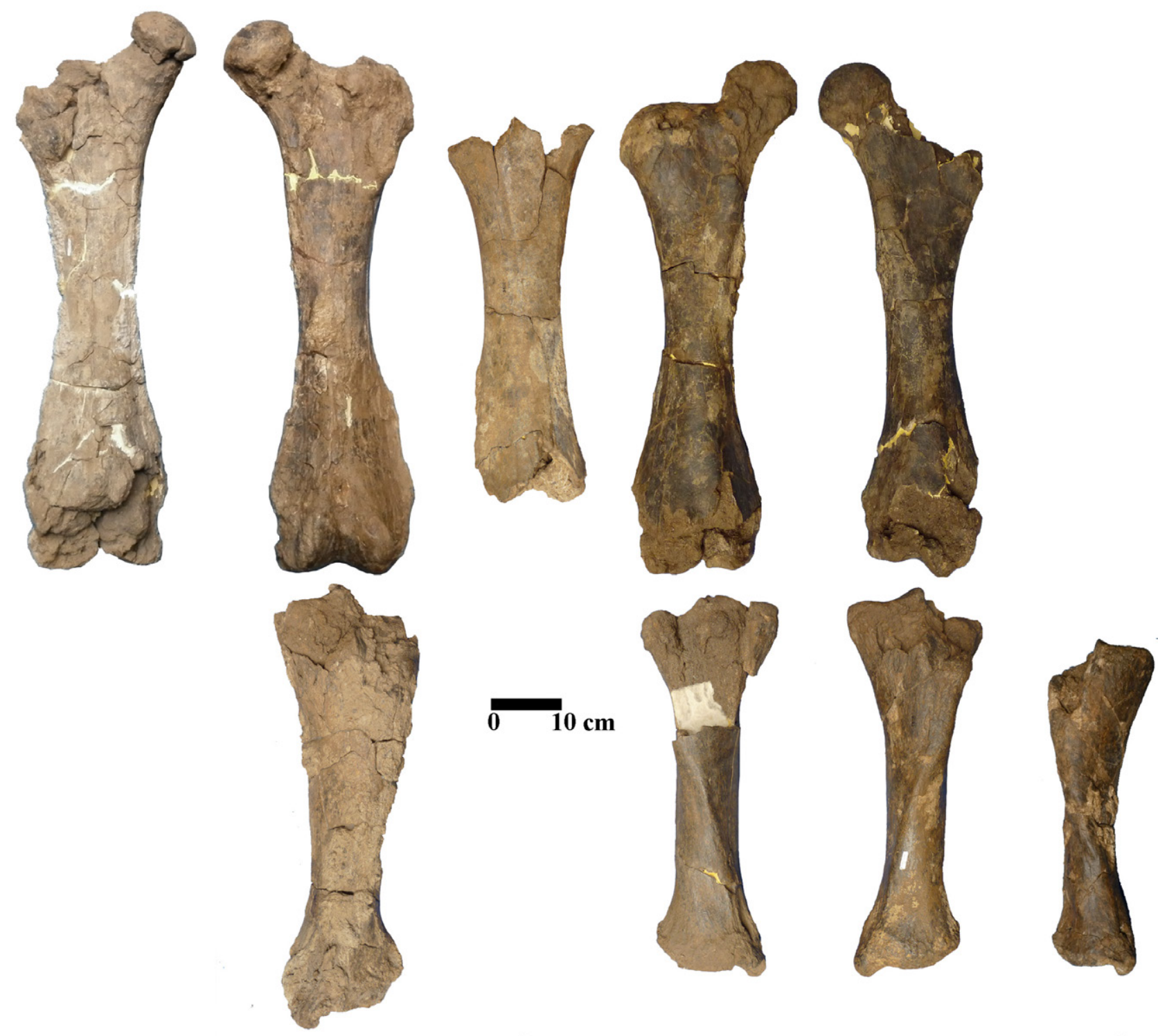

a

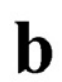

c

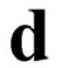

Figura 8. Individuos de la muestra, representados por fémures y tibias. a) Individuo 1 (DP-5800, 5804, 5807); b) individuo 2 (DP-5802); c) individuo 3 (DP-5801,5803, 5806, 5808); d) individuo 4 (DP-5805).

individuo 3 está constituido por dos fémures y dos tibias con mediano estado de conservación (Figura 8c); el individuo 4 está representado por la tibia de menor tamaño y mejor estado de conservación (Figura 8d).

Las diferencias de tallas en el mismo tipo de hueso de ejemplares adultos en esta población fueron interpretadas como una expresión de dimorfismo sexual, asignando los ejemplares más grandes y robustos a machos y los individuos pequeños y gráciles, a las hembras (Tabla 2).

Por su parte, el análisis bivariante del $\mathrm{M}_{3}$ (Figura 9) sugiere que la pieza completa de Colima pertenece a un individuo de tamaño intermedio entre los hallados en el país, comparable con el material de Tzintzuntzan en Michoacán, con uno de los dos molares de Rancho Gerardo en Puebla, con el de Chapala-Zacoalco en Jalisco y con otros sin localidad precisa (Montellano-Ballesteros, 2002; Alberdi y Corona-M., 2005; Robles-Camacho et al., 2010). El molar de Colima es $32 \%$ más corto que el ejemplar más largo conocido, procedente de Chile (Frassinetti y Alberdi, 2000) y $20 \%$ más corto que el molar más largo de México y que pertenece al ejemplar tipo de Tequixquiac (Alberdi y Corona-M., 2005).

\subsection{Comparación}

4.4.1. Comparación morfológica del esqueleto postcraneal

El húmero recuperado en Colima tiene rasgos gonfoterinos (más parecido al de los gonfoterios que al del 


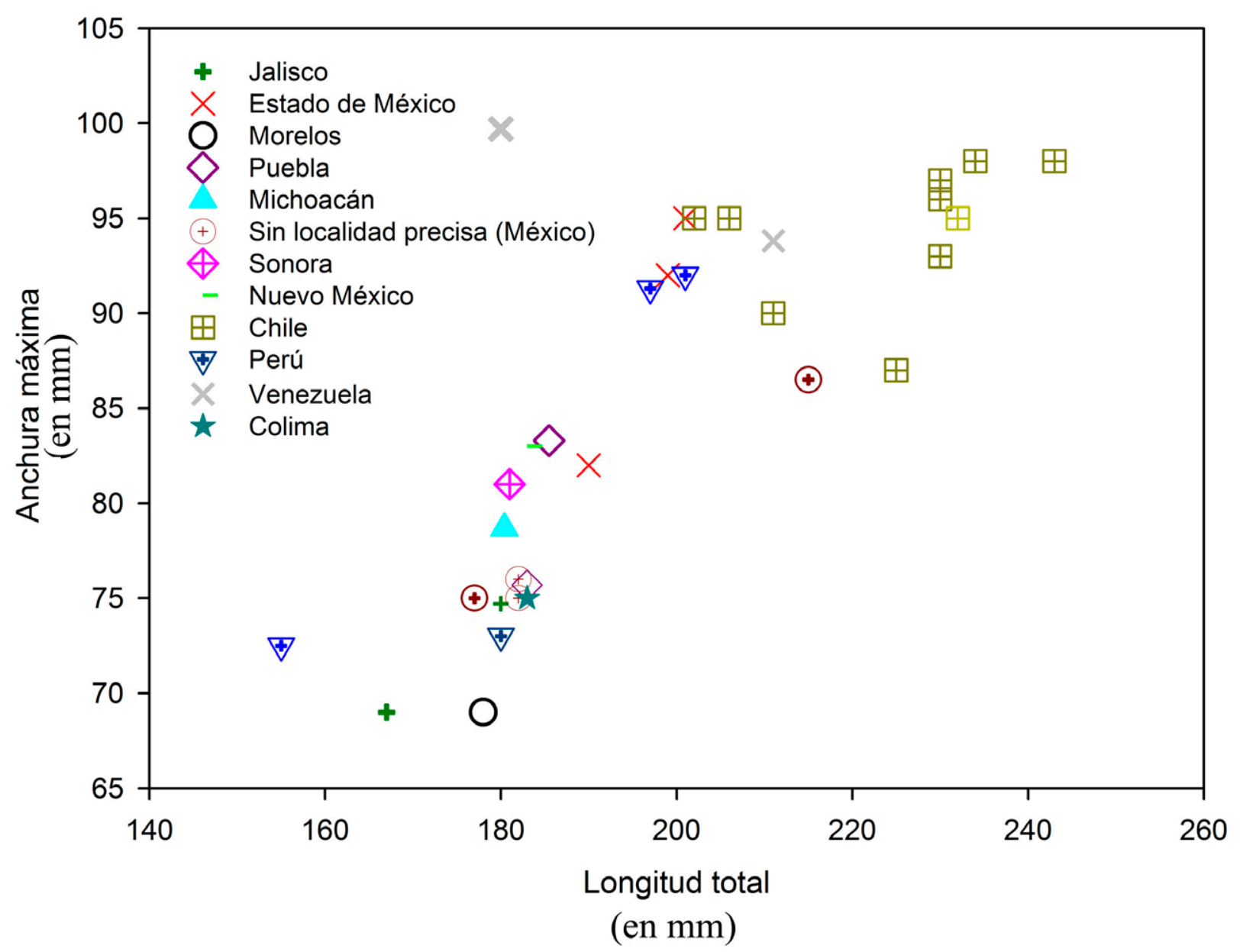

Figura 9. Relación longitud total y anchura máxima de terceros molares inferiores pertenecientes a diferentes ejemplares de gonfoterio del género Cuvieronius.

mamut o al del mastodonte). Se diferencia del mastodonte y del mamut, porque el surco musculoespiral es más profundo en Cuvieronius y en aquéllos no, por tener las crestas deltoidea y supracondiloidea muy bajas; de hecho, la cresta deltoidea está poco desarrollada en el mastodonte y es casi ausente en el mamut. El húmero del mastodonte en vista lateral es más ancho en su extremo proximal en relación con el resto del hueso, y por ello adquiere una forma cónica invertida, como ocurre en el gonfoterio de Colima. El húmero de Rhynchotherium y Gomphotherium, respecto al de Cuvieronius, es más ancho en la epífisis distal y tiene un notable desarrollo de la cresta supracondiloidea (Pasenko, 2007; Göhlich, 2010); además, en Rhynchotherium la cresta deltoidea está más expandida en su porción media y el borde medial es más cóncavo. El húmero de Stegomastodon tuvo una morfología variable, ya que la pieza derecha es muy parecida a la de Cuvieronius, mientras que en la pieza izquierda, la cresta deltoidea está muy expandida y la cresta supracondiloidea se inserta en la diáfisis en un ángulo más abierto; el daño en la tuberosidad lateral en el material de Colima impide verificar si es de mayor tamaño que en Stegomastodon, como observaron Lucas et al. (2011), carácter que sí se observa en el húmero del Cuvieronius recuperado en Ecuador (Boule y Thevenin, 1920).

La ulna de Cuvieronius comparte con la de Mammuthus, Gomphotherium, Rhynchotherium, y posiblemente Stegomastodon, un proceso anconeano ancho. En Rhynchotherium la pieza es además muy ancha. El proceso olecranon en Cuvieronius es vertical pero en Mammut y en Mammuthus es inclinado; esta área está dañada en los otros géneros de gonfoterios, o no se ilustra ni se describe. En Stegomastodon el borde posterior de la diáfisis describe una amplia curva cóncava, mientras que en Cuvieronius la zona de la curvatura se restringe a la porción proximal y el resto del borde es recto.

Respecto al hueso innominado, el ilion es amplio en todos los géneros de proboscidios. El isquion es ancho y el pubis es corto en Mammuthus y Mammut, lo que da lugar a un foramen obturador pequeño; en cambio, en los gonfotéridos en los que se ha conservado el innominado, el isquion y el pubis son delgados y este último también es alargado, lo que ocasiona que el foramen obturador sea amplio.

El fémur del gonfoterio también es típicamente gonfoterino y se diferencia de los de Mammut y Mammuthus porque la cabeza articular no sobrepasa notoriamente al 
trocánter. La diáfisis en Cuvieronius, Rhynchoherium y Stegomastodon se adelgaza en su parte media, contrario a lo que sucede en Mammut, Mammuthus y Gomphotherium, cuyos bordes son más rectos, aunque en éste último hay una pequeña constricción en la mitad proximal. En Rhynchotherium, la cabeza articular es amplia y prácticamente carece de cuello, mientras que el trocánter mayor es más grande (en Cuvieronius, la cabeza es poco amplia, el cuello angosto y corto y el trocánter está poco marcado y no es tan amplio). En general, el fémur de Cuvieronius es más semejante al de Gomphotherium y Stegomastodon por la constricción media de la diáfisis (aunque en Gomphotherium está desplazada hacia la porción proximal) y la cabeza baja.

La tibia de Cuvieronius se diferencia de la de Mammut y de Mammuthus en la cresta tibial, que en uno es poco evidente y en el otro evidente sólo proximalmente (la del gonfoterio de Colima es evidente a todo lo largo de la pieza). La epífisis distal en Mammut es más o menos cuadrada, mientras que en Cuvieronius es redondeada. En Stegomastodon, en cambio, se presenta un maléolo muy prominente y la cresta tibial está menos marcada, aunque también está a todo el largo de la pieza. En Rhynchotherium, el maléolo también es más prominente que en Cuvieronius; el comportamiento de la cresta no ha sido descrito. En Gomphotherium el maléolo es muy bajo, como en Cuvieronius, aunque la cresta no es perceptible. La tibia en Cuvieronius comparte con los otros proboscidios la característica de tener el cóndilo medial más alto que el lateral y la espina intercondilar baja.

La tróclea del astrágalo es redondeada en Cuvieronius y Mammuthus, mientras que en Mammut, Gomphotherium y Stegomastodon es cuadrangular; Mammut presenta una protuberancia posterior no observada en los otros proboscidios. Ventralmente, la forma de las carillas articulares y la posición del surco intermedio, lo hacen indistinguible del astrágalo de Stegomastodon; en los otros géneros se presentan formas y disposiciones diferentes. No se conoce la apariencia de este hueso en Rhynchotherium.

El calcáneo de Cuvieronius presenta la tuberosidad alargada y sin protuberancias, como en Stegomastodon; la tuberosidad en otros proboscidios es cuadrangular (carece de cuello: Mammuthus, Rhynchotherium), muy redonda (Mammut americanum) o variable (Gomphotherium: de muy alargada a muy redondeada; en ambos casos con cuello). El sustentáculo en Cuvieronius es corto, poco prominente, mientras que en los otros proboscidios está muy bien diferenciado, separado inclusive del cuerpo del calcáneo por una muesca (en Mammut y Mammuthus) y en Gomphotherium es variable. Además, el sustentáculo en Mammuthus está dirigido posterad, mientras que en los demás proboscidios está dirigido mediad. Esta pieza es más

Tabla 3. Medidas comparativas de huesos largos de distintas familias de proboscidios reportadas en la literatura.

\begin{tabular}{|c|c|c|c|c|c|c|c|c|c|c|c|c|c|c|c|c|c|c|c|}
\hline & \multicolumn{4}{|c|}{ Fémur } & \multicolumn{4}{|c|}{ Tibia } & \multicolumn{4}{|c|}{ Húmero } & \multicolumn{4}{|c|}{ Ulna } & \multirow{2}{*}{$\begin{array}{c}\text { Pata } \\
\text { trasera } \\
\quad \text { L } \\
\end{array}$} & \multirow{2}{*}{$\begin{array}{c}\text { Pata } \\
\text { delantera } \\
\mathbf{L} \\
\end{array}$} & \multirow[t]{2}{*}{ Fuente } \\
\hline & $\mathbf{L x}$ & Ap & Ad & $\mathbf{A m}$ & $\mathbf{L x}$ & Ap & Ad & Am & $\mathbf{L x}$ & Ap & Ad & $\mathbf{A m}$ & $\mathbf{L x}$ & Ap & Ad & Am & & & \\
\hline & \multicolumn{19}{|c|}{ Gomphotheriidae } \\
\hline $\begin{array}{l}\text { Gomphotherium } \\
\text { aff. G. } \\
\text { steinheimense } \\
\text { (Alemania) }\end{array}$ & 1200 & 380 & 285 & 159 & 775 & 260 & 195 & 106 & 1010 & -290 & 222 & 144 & 930 & 236 & 166 & 121 & 1975 & 1940 & Göhlich, 1998 \\
\hline $\begin{array}{c}\text { Gomphotherium } \\
\text { angustidens } \\
\text { (Francia) }\end{array}$ & ----- & ---- & ----- & ----- & ---- & ---- & ----- & ---- & 670 & ---- & 155 & 100 & 630 & ----- & 65 & 78 & ---- & 1300 & Tassy, 1977 \\
\hline Rhynchotherium & 905 & 413 & ---- & ---- & 657 & ---- & 204 & 111 & 902 & 334 & & 152 & 905 & 277 & ---- & 143 & 1830 & 1767 & $\begin{array}{l}\text { Pasenko, } \\
2007\end{array}$ \\
\hline $\begin{array}{c}\text { Stegomastodon } \\
\text { primigenius }\end{array}$ & 1095 & 392 & 392 & 195 & 735 & 263 & 228 & ---- & 945 & 340 & 440 & 116 & 822 & ----- & ----- & ----- & 1562 & 1807 & $\begin{array}{l}\text { Lucas et al., } \\
\quad 2011\end{array}$ \\
\hline $\begin{array}{l}\text { Cuvieronius } \\
\text { tropicus }\end{array}$ & 975 & 340 & 212 & 152 & ---- & ----- & -158 & -89 & 675 & 174 & 173 & 105 & ----- & ----- & ---- & ----- & ----- & ----- & $\begin{array}{l}\text { Montellano- } \\
\text { Ballesteros, } \\
\quad 2002\end{array}$ \\
\hline \multirow[t]{2}{*}{$\begin{array}{c}\text { Cuvieronius } \\
\text { hyodon }\end{array}$} & 902 & 259 & 205 & 125 & 578 & 196 & 178 & 93.6 & 560 & 174 & 184 & 84 & 725 & ---- & 138 & 120 & 1480 & $1285^{*}$ & Este trabajo \\
\hline & \multicolumn{19}{|c|}{ Mammutidae } \\
\hline $\begin{array}{l}\text { Mammut } \\
\text { americanum }\end{array}$ & 1060 & ---- & ----- & ----- & 710 & ----- & 205 & ---- & 860 & 282 & ----- & ----- & 775 & ---- & 167 & ---- & 1770 & 1635 & Miller, 1987 \\
\hline & \multicolumn{19}{|c|}{ Elephantidae } \\
\hline $\begin{array}{l}\text { Mammuthus } \\
\text { primigenius }\end{array}$ & 1200 & ---- & ---- & ---- & 675 & ---- & ---- & ---- & 1000 & ---- & ---- & ---- & 770 & ---- & ---- & ---- & 1875 & 1770 & Haynes, 1991 \\
\hline $\begin{array}{l}\text { Loxodonta } \\
\text { africana }\end{array}$ & 1330 & ---- & ---- & ---- & 785 & ---- & ---- & ---- & 1110 & ---- & ---- & ----- & 810 & ---- & ---- & ---- & 2115 & 1920 & \\
\hline
\end{tabular}

Lx: longitud máxima, Ap: anchura de la epífisis proximal, Ad: anchura de la epífisis distal, Am: anchura más pequeña de la diáfisis. L: longitud obtenida de la suma de sus elementos (longitudes del húmero y de la ulna: longitudes del fémur y de la tibia). Las medidas están dadas en milímetros. Medidas incompletas entre paréntesis. Los guiones indican la ausencia del dato. * Asumiendo que los huesos pertenecen al mismo individuo. 
parecida a la de Rhynchotherium y Stegomastodon.

\subsubsection{Comparación de talla}

La diferencia más patente en los huesos largos de los individuos adultos de diferentes proboscidios es desde luego la talla, siendo Cuvieronius, en términos generales, el proboscidio más bajo de todos (Tabla 3 ).

Respecto del individuo de Cuvieronius de mayor talla, los huesos de la extremidad posterior de un mamut son de $17 \%$ (tibia) a $33 \%$ (fémur) más grandes, mientras que las diferencias en ambas piezas son semejantes con el mastodonte y el gonfoterio de Jalisco (Mammut: $20 \%$ fémur, $23 \%$ tibia; Stegomastodon: $21 \%$ fémur, $27 \%$ tibia); en cambio, el fémur en Rhynchotherium es apenas mayor que el de Cuvieronius (0.003 \%), aunque la diferencia con la tibia es mayor (14\%) (Tabla 3). La comparación con los huesos disponibles de la extremidad anterior, muestra una mayor diferencia en el húmero que en la ulna (Mammuthus: $78 \%$ húmero; $6 \%$ ulna; Mammut: $53 \%$ húmero, $6 \%$ ulna; Stegomastodon: $69 \%$ húmero, $13 \%$ ulna; Rhynchotherium: $61 \%$ húmero, $25 \%$ ulna; Gomphotherium: $20 \%$ húmero, $15 \%$ ulna), lo que quizá pueda deberse a que estos huesos en el material de Colima quizá no pertenezcan a un mismo individuo.

A través de la Tabla 3 y de los porcentajes obtenidos se observa que la baja estatura de Cuvieronius en el miembro posterior se debe a que el fémur y la tibia son más cortos y que Stegomastodon tiene una talla semejante a Cuvieronius. En cuanto a las diferencias extremas obtenidas en el húmero, una posible explicación es que el ejemplar de Colima pertenezca a una hembra; la semejanza de tamaño con el del ejemplar francés de Gomphotherium, sugiere que este último también pertenezca a una hembra.

\section{Discusión}

Si bien todavía se requiere obtener conjuntos de varios molares de una misma localidad, preferentemente asociados a mandíbulas relativamente completas, para establecer la variación poblacional de los lófidos (número y forma) y sus implicaciones taxonómicas, es de notar que la variación en el contorno de la figura oclusal ha sido poco destacada, el cual en Cuvieronius puede ser simple (bordes de esmalte sin pliegues: mayoría de los casos) a complejo (bordes de esmalte plegados, pero sin llegar a la complejidad que se presenta en el género Stegomastodon; esta característica ha sido hallada en ejemplares en Sonora [Lucas y González de León, 1997] y Michoacán [Robles-Camacho et al., 2010]); el material de Colima es, en este aspecto, del tipo general o simple.

Con respecto al postcraneal, la comparación anatómica de los principales huesos largos y tarsales indica que es posible diferenciar a las tres familias a través de estos elementos óseos, apoyando, en lo general, las observaciones de Boule y Thevenin (1920) hechas para gonfotéridos (de
Sudámerica y Europa) y elefántidos (formas recientes y fósiles); también mostró que la morfología ósea de Cuvieronius es muy semejante a la de Stegomastodon, confirmando así los resultados de los análisis filogenéticos previos basados en caracteres morfológicos dentales, craneales y postcraneales, que concluyen que ambos géneros están cercanamente emparentados (Shoshani, 1996; Prado y Alberdi, 2008).

A pesar de ese parecido, sí existen varias características exclusivas de Cuvieronius, pero para observarlas adecuadamente en la mayoría de los casos se requiere tener la pieza completa. La morfología del húmero y la tibia de la población colimense resultaron ser bastante uniformes con la población de Cuvieronius de Tarija en Bolivia; en cambio, la ulna del material sudamericano tiene una marcada concavidad en el borde posterior de la diáfisis y un grosor mayor del hueso, mientras que el fémur está ligeramente más ensanchado en sus porciones distal y proximal y la fíbula presenta los bordes anterior y posterior rectos. La población de Bolivia es considerada por algunos investigadores como una especie de Cuvieronius diferente a C. hyodon (e.g. Ficcarelli et al., 1995), y más aún, la morfología de la ulna (ver Ficcarelli et al., 1995) en Haplomastodon (Stegomastodon sensu lato) es la misma que la de la pieza de Tarija, lo que sugiere, apoyado en que la morfología debe ser conservativa, la mezcla de materiales de distintos géneros en esas localidades sudamericanas. Otros autores consideran que no hay claras diferencias entre estas formas y que todo es parte de la variabilidad poblacional que se da en los gonfoterios (Alberdi, com.pers. 2013), ya que la variabilidad craneal, mandibular y dental (defensas) también ha sido relacionada al dimorfismo sexual (Boule y Thevenin, 1920). Con respecto a los otros géneros de proboscidios, el húmero y la tibia fueron las piezas más distintivas para reconocer a Cuvieronius.

Por otro lado, el tamaño de los huesos largos sugiere que la población colimense es de pequeñas proporciones, aún más que uno de los ejemplares de Rancho Gerardo en Puebla, localidad en donde también se recuperó un molar del mismo tamaño que el de Colima, pero también otro más grande. Esta posible correspondencia en tamaño de molares y huesos largos, sugeriría por tanto que los individuos de Chapala en Jalisco (basados en molares), habrían sido de una talla aproximada al de los gonfoterios de Colima, en cambio, los molares de Tequixquiac, Estado de México, sugieren que habrían sido animales de mayor talla (molares de $201 \mathrm{~mm}$ x 95 mm y 199 mm x 92 mm; ver Figura 17).

El dimorfismo sexual en proboscidios, tanto de los fósiles como de los actuales, ha sido constatado por diferentes autores para otras familias de proboscidios (Haynes, 1991; Averianov, 1996; Palombo y Villa, 2003) así como para el género Gomphotherium (Tassy, 1996). También ha sido sugerido para otros gonfoterios en distintas partes del mundo (Boule y Thevenien, 1920; Laurito, 1988; Ficcarelli et al., 1993; Geraads et al., 2005; Gölihch, 2007; Konidaris y Koufos, 2009; Ferretti, 2010). En la mayoría 
de los gonfoterios, esa diferenciación sexual está basada exclusivamente en piezas dentales, por lo que el material de Colima proporciona ahora una evidencia complementaria para Cuvieronius.

La presencia de Cuvieronius en Colima era esperada, dado los registros en los estados colindantes (Michoacán y Jalisco) y su presencia tanto en el norte como en el sur de México. En relación a la distribución general de Cuvieronius, si bien los registros han sido más numerosos en los estados que limitan con el Océano Pacífico, situación que ha motivado la hipótesis de un corredor de dispersión occidental en México (Alberdi y Corona-M., 2005), ahora empiezan a ser cada vez más los hallazgos en la vertiente atlántica, por lo que la dispersión del grupo se habría efectuado utilizando las tierras bajas de ambas vertientes, a semejanza de lo que se observa en especies actuales de afinidad tropical (como el tlacuache, el armadillo, el puercoespín: Webb, 1976). La ubicación de la Guayabilla, un área tropical muy próxima a la costa y asociada a una vía fluvial para penetrar a las tierras interiores más templadas, muestra la versatilidad de ambientes y paisajes que este género fue capaz de colonizar en México, aunque en su dispersión hacia Sudamérica habría sido desplazado por otros gonfoterios (Stegomastodon waringi y S. platensis) hacia zonas templadas y de mayor altitud (Laurito, 1988; Prado et al., 2005).

\section{Conclusión}

Los restos postcraneales de al menos cuatro individuos del gonfoterio Cuvieronius hyodon, los primeros que se documentan para el estado de Colima, muestran una clara afiliación gonfoterina, pero también rasgos propios que permiten diferenciarlos fácilmente de los dos géneros de gonfoterios más antiguos (Rhynchotherium y Gomphotherium); no así del género Stegomastodon, con el cual comparte una mayor semejanza derivada de su mayor parentesco filogenético. Rasgos tales como los bordes rectos del húmero, particularmente el borde medial en su extremo proximal, y el maléolo medial de la tibia poco prominente, son únicos de Cuvieronius.

El análisis bivariante de los $\mathrm{M}_{3}$ mostró que la población colimense de Cuvieronius es de tamaño intermedio entre aquellas del centro del país y pequeño en relación a otros del resto del continente. Además, el tamaño de los huesos de las extremidades ubica al gonfoterio Cuvieronius hyodon como un proboscidio de talla pequeña, más pequeña que la de su pariente más cercano (Stegomastodon), aunque queda por evaluar la variación al interior de los géneros para determinar si las diferencias de talla son debidas a diferencias taxonómicas o al dimorfismo sexual y que grado de solapamiento tienen. Asimismo, el contorno de la figura oclusal es de tipo simple.

Finalmente, la distribución de Cuvieronius en el país cubrió ambas vertientes, por lo que la dispersión de este taxón habría sido preferentemente a través de las tierras costeras.

\section{Agradecimientos}

Deseamos agradecer a María Teresa Alberdi y a Víctor Manuel Bravo Cuevas, la revisión del texto y cuyos acertados comentarios y sugerencias permitieron mejorarlo. Agradecemos la valiosa ayuda de la Biól. Tania Lozano Ramos, que en su momento colaboró en la limpieza de parte del material.

\section{Referencias}

Álvarez, T., 1982, Restos de mamíferos recientes y pleistocénicos procedentes de las grutas de Loltún, Yucatán, México: Instituto Nacional de Antropología e Historia, Departamento de Prehistoria, Cuadernos de Trabajo, 26,7-35.

Alberdi, M.T., Corona-M., E., 2005, Revisión de los gonfoterios del Cenozoico tardío de México: Revista Mexicana de Ciencias Geológicas, 22, 246-260.

Alberdi, M.T., Juárez-Woo, J., Polaco, O., Arroyo-Cabrales, J. 2009. Description of the most complete skeleton of Stegomastodon (Mammalia, Gomphotheriidae) recorded for the Mexican Late Pleistocene: Neues Jahrbuch für Geologie und Paläontologie, Abhandlungen, 251, 239-255.

Alberdi, M.T., Prado, J.L., Salas, R., 2004, The Pleistocene Gomphotheriidae (Proboscidea) from Peru: Neues Jahrbuch für Geologie und Paläontologie, Abhandlungen, 231, 423-452.

Arroyo-Cabrales, J., Polaco, O.J., Johnson, E., 2002, La mastofauna del Cuaternario tardío en México, en Montellano-Ballesteros, M., Arroyo-Cabrales, J. (coords.), Avances en los estudios paleomastozoológicos en México: México, D. F., Instituto Nacional de Antropología e Historia, 103-123.

Arroyo-Cabrales, J., Polaco, O.J., Laurito, C., Johnson, E., Alberdi, M.T., Valerio Zamora, A.L., 2007, The proboscideans (Mammalia) from Mesoamerica: Quaternary International, 169-170, 17-23.

Arroyo-Cabrales, J., Polaco, O.J., Johnson, E., Ferrusquía-Villafranca, I., 2010, A perspective on mammal biodiversity and zoogeography in the late Pleistocene of Mexico: Quaternary International, 212,187197.

Averianov, A.O., 1996, Sexual dimorphism in the mammoth skull, teeth and long bones, en Shoshani, J., Tassy, P. (eds), The Proboscidea: evolution and palaeoecology of elephants and their relatives: Oxford, Oxford University, 260-267.

Böese, E., 1905, Reseña acerca de la geología de Chiapas y Tabasco: Boletín del Instituto Geológico, 20, 1-116.

Boule, M., A. Thevenin, 1920, Mammiferes fossils de Tarija. Mission Scientifique. G. de Créqui-Montfort et E. Sénéchal de la Grange. $78 \mathrm{p}$.

Cabrera, A., 1929, Una revisión de los mastodontes argentinos: Revista del Museo de la Plata, 32, 61-144.

Castillo, C.M., Cabral, M.A., Carranza-Castañeda, O., 1996, Vertebrados fósiles del estado de Hidalgo Fossil vertebrates from State of Hidalgo: México D.F., Universidad Autónoma del Estado de Hidalgo, $127 \mathrm{p}$.

Chávez-Aponte, E.O., Alfonso-Hernández, I., Carrillo-Briceño, J.D., 2008, Morfología dentaria de los gonfoterios de la localidad de Muaco, estado Falcón, Venezuela: Interciencia, 33(10), 771-775.

Cope, E.D., 1884, The extinct Mammalia of the Valley of Mexico: Proceedings of the American Philosophical Society of Philadelphia, 22, 1-21. 
Cope, E.D., 1893, Description of a lower jaw of Tetrabelodon shepardii Leidy: Proceedings of the Academy of Natural Sciences of Philadelphia, 45(2), 202-204.

Corona-M., E., Alberdi, M.T., 2006, Two new records of Gomphotheriidae (Mammalia: Proboscidea) in Southern México and some biogeographic implications: Journal of Paleontology, 80, 357-366.

DeBlase, A.F., Martin, R.E., 1974, A manual of Mammalogy with keys to families of the World: Iowa, Brown Company Publishers, $436 \mathrm{p}$.

Del Castillo, A., 1869, Clasificación y datos sobre los mamíferos fósiles encontrados en el Valle de México: Deutsche Geologische Gesellschaft, Zeitschrift, 21, 479-480.

Downs, T., 1958, Fossil vertebrates from Lago de Chapala, Jalisco, México (resumen), en Congreso Geológico Internacional: México D.F., México, (A. García Rojas, E. J. Guzmán, J. González Reyna, F. Mina Uhink, S. Figueroa y M. Maldonado-Koerdell, eds.), 75-77.

Eaton, G.F., 1905, Occurrence of Mastodon humboldtii in Northern Mexico: The American Journal of Science, Fourth Series, 19, 330.

Felix, J., Lenk, H., 1891, Uebersicht über die geologischen Verhältnisse des mexicanischen Staates Puebla: Palaeontographica, 117-139.

Ferretti, M.P., 2010, Anatomy of Haplomastodon chimborazi (Mammalia, Proboscidea) from the Late Pleistocene of Ecuador and its bearing of the phylogeny and systematics of South American gomphotheres: Geodiversitas, 32, 663-721.

Ferrusquía-Villafranca, I., Arroyo-Cabrales, J., Martínez-Hernández, E., Gama-Castro, J., Ruíz-González, J., Polaco, O.J., Johnson, E., 2010, Pleistocene mammals of Mexico: a critical review of regional chronofaunas, climate change response and biogeographic provinciality: Quaternary International, 217, 53-104.

Ficcarelli, G., Borselli, V., Moreno-Espinosa, M., Torre, D., 1993, New Haplomastodon finds from the late Pleistocene of northern Ecuador: Geobios, 26, 231-240.

Ficcarelli, G., Borselli, V., Herrera, G., Moreno-Espinosa, M. Torre, D. 1995. Taxonomic remarks on the South American mastodons referred to Haplomastodon and Cuvieronius: Geobios, 28, 745-756.

Fischer, G., 1814, Zoognosia. Tabulis synopticis illustrate: Moscow, Typis Nicolai Sergeidis Vsevolozsky, 3, 1-694.

Frassinetti, D., Alberdi, M.T., 2000, Revisión y estudio de los restos fósiles de mastodontes de Chile (Gomphotheriidae): Cuvieronius hyodon, Pleistoceno Superior: Estudios Geológicos, 56, 197-208.

Freudenberg, W., 1922, Die Säugertier Fauna des Pliocäns und Postpliocäns von Mexiko, II, Mastodonten und Elefanten: Geologische und Paläontogische Abhandlungen, 14, 103-176.

García, E., 1973, Modificación al sistema de clasificación climática de Köppen: México D.F., Universidad Nacional Autónoma de México, $246 \mathrm{p}$.

García-Zepeda, M.L., 2006, Nouvi dati paleontologici dalla depressione lacustre di Cuitzeo, Michoacan, Messico: Firenze. Italia, Universitá degli Studi di Firenze, tesis doctoral, 115 p.

Geraads, D., Kaya, T., Mayda, S., 2005, Late Miocene large mammals from Yulafli, Thrace region, Turkey, and their biogeographic implications: Acta Palaeontologica Polonica, 50, 523-544.

Göhlich, U.B., 1998, Elephantoidea (Proboscidea, Mammalia) aus dem Mittel- und Obermiözan der Oberen SüBwassermolasse Süddeutschlands: Odontologie und Osteologie: Münchner Geowissenschaftliche Abhandlungen, A36: 1-245.

Göhlich, U.B., 2007, Gomphotheres (Proboscidea, Mammalia) from the Early-Middle Miocene of Central Mongolia: Annalen des Naturhistorischen Museums in Wien, 108A: 271-289.

Göhlich, U.B., 2010, The Proboscidea (Mammalia) from the Miocene of Sandelzhausen (southern Germany): Paläontologische Zeitschrift, 84: 163-204

Haynes, G., 1991, Mammoths, mastodonts and elephants: biology, behavior, and the fossil record: New York, Cambridge University Press, $412 \mathrm{p}$.

Instituto Nacional de Geografía, Estadística e Informática (INEGI), 1981, Síntesis Geográfica de Colima: México, D. F., Coordinación General de los Servicios Nacionales de Estadística, Geografía e
Informática, $131 \mathrm{p}$.

Jiménez-Hidalgo, E., Guerrero-Arenas, R., Macfadden, B.J., CabreraPérez, L., 2011, The late pleistocene (Rancholabrean) Viko vijin local fauna from La Mixteca Alta, Northwestern Oaxaca, Southern Mexico: Revista Brasileira de Paleontología, 14, 15-28.

Klein, G.R., Cruz-Uribe, K., 1984, The analysis of animal bones from archaeological sites: Chicago, The University of Chicago Press, $266 \mathrm{p}$.

Konidaris, G.E., Koufos, G.D., 2009, The Late Miocene Mammal Faunas of the Mytilinii Basin, Samos Island, Greece: New Collection: Beiträge zur Paläontologie, 31:139-155.

Lambert, W.D., 1996, The biogeography of the gomphotheriid proboscideans of North America, en Shoshani, J., Tassy, P. (eds.), The Proboscidea: Evolution and Palaeoecology of Elephants and their relatives: Oxford, Oxford University Press, 143-148.

Laurito, C.A., 1988, Los proboscideos fósiles de Costa Rica y su contexto en la América Central: Vínculos, 14, 29-58.

Lucas, G.S., 2003, Pleistocene proboscideans from Lago de Chapala, Jalisco, Mexico: Current Research in the Pleistocene, 20, 105-107.

Lucas, G.S., González-León, C.M., 1997, Cuvieronius (Mammalia, Proboscidea) de Oquitoa, Sonora: Geología del Noroeste, 2, 12-13.

Lucas, G.S., Aguilar, R.H., Spielman, J.A., 2011, Stegomastodon (Mammalia, Proboscidea) from the Pliocene of Jalisco, Mexico and the species-level taxonomy of Stegomastodon: Bulletin of the New Mexico Museum of Natural History and Science, 53: 517-553.

Mazo-Pérez, A.N., 1995, Proboscideos I: bariterios, meriterios, dinoterios y mastodontes (Superfamilias: Baritherioidea, Moeritherioidea, Deinotherioidea y Elephantoidea, excepto Elephantidae), en Meléndez, B. (comp.), Paleontología: mamíferos fósiles ( $2^{\mathrm{a}}$ parte): Madrid, Paraninfo, 251-309.

Mead, J.I., Baez, A., Swift, S.L., Carpenter, M.C., Hollenshead, M., Czaplewski, N.J., Steadman, D.W., Bright, J., Arroyo-Cabrales, J., 2006, Tropical marsh and savanna of the late Pleistocene in Northeastern Sonora, Mexico: The Southwestern Naturalist, 51, 226-239.

Miller, W.E., 1987, Mammut americanum, Utah's fisrt record of the American mastodon: Journal of Paleontology, 61, 168-183.

Miller, W.E., Carranza-Castañeda, O., 1984, Late Cenozoic mammals from central Mexico: Journal of Vertebrate Paleontology, 4, 216-236.

Montellano-Ballesteros, M., 2002, New Cuvieronius findings from the Pleistocene of central Mexico: Journal of Paleontology-, 76, 578583.

Ochoterena, F.H., Silva-Bárcenas, A., 1970, Cuvieronius arellanoi sp. n., mastodonte del Pleistoceno del estado de Oaxaca: Paleontología Mexicana, 33, 5-22.

Olsen, S.J., 1979, The American mastodon and the woolly mammoth: Papers of the Peabody Museum of Archaeology and Ethnology, $56,1-44$.

Osborn, H.F., 1923, New subfamily, generic, and specific stages in the evolution of the Proboscidea: American Museum Novitates, 99, 1-4.

Osborn, H.F., 1936, Proboscidea: a monograph of the discovery, evolution, migration, and extinction of the mastodons and elephants of the world. Vol. I: Moeritherioidea, Deinotherioidea, Mastodontoidea: New York, The American Museum Press, I, 1-804.

Osborn, H.F., 1942, Proboscidea: a monograph of the discovery, evolution, migration, and extinction of the mastodons and elephants of the world. Vol. II: Stegodontoidea, Elephantoidea: New York, The American Museum Press, II, 805-1675.

Palombo, M.R., Villa, P., 2003, Sexually dimorphic characters of Elephas (Palaeoloxodon) antiquus from Grotte Santo Stefano (Viterbo, Central Italy), en Reumer, J.W.F., De Vos, J., Mol, D. (eds), Advances in Mammoth Research: Proceedings of the Second International Mammoth Conference: Rotterdam, Deinsea, 9: 293-315.

Pasenko, R.M, 2007, Rhynchotherium falconeri (Proboscidea, Gomphotheridae) from 111 Ranch, Southeastern Arizona with a discussion of the genus: Journal of Vertebrate Paleontology, 27, 195-203. 
Peña-Serrano, J., Lozano-Ramos, T., Aguilar, F.J., Miranda, F., 2004, Hallazgos paleontológicos recientes en la región de CórdobaOrizaba, Veracruz, México (resumen), en IX Congreso Nacional de Paleontología: Tuxtla Gutiérrez, Chiapas, México, Sociedad Mexicana de Paleontología, 46.

Peña-Serrano, J., Miranda Flores, F.A., 2009, Presencia de restos de mastofauna pleistocénica en la región de las grandes montañas del estado de Veracruz, México (resumen), en XI Congreso Nacional de Paleontología: Juriquilla, Querétaro, México, Sociedad Mexicana de Paleontología, 58.

Pichardo del Barrio, M, 1960, Proboscideos fósiles de México, una revisión: Instituto Nacional de Antropología e Historia, Colección Científica, 4,1-63.

Polaco, O.J., 1988, Restos óseos de animales procedentes del Cerro del Pino, orden 9-87: México D.F., México, Laboratorio de Paleozoología, Subdirección de Laboratorios y Apoyo Académico, Instituto Nacional de Antropología e Historia, Informe de trabajo Z-416, 1 p.

Polaco, O.J. 2002, Los proboscidios de México, en González-González, A.H., De Stefano-Farías, A. (eds.), Fósiles de México: Coahuila, una ventana a través del tiempo: Saltillo, Gobierno del Estado de Coahuila, 179.

Polaco, O.J. 2008, Informe de la comisión al Centro Regional INAH Guanajuato: México D.F., México, Laboratorio de Paleozoología, Subdirección de Laboratorios y Apoyo Académico, Instituto Nacional de Antropología e Historia, 2 p.

Polaco, O.J., Aguilar, F.J., 1999, Fauna fósil procedente de Vichinchijol, Ébano San Luis Potosí, orden 9-99: México D.F., México, Laboratorio de Paleozoología, Subdirección de Laboratorios y Apoyo Académico, Instituto Nacional de Antropología e Historia, Informe de trabajo Z-512, $15 \mathrm{p}$.

Polaco, O.J., González, A.H., Rojas, C., 2002, Se trata de un gonfoterio: revisión sobre el hallazgo en un cenote de Yucatán: National Geographic en español II(5): Geográfica, v-vi.

Prado, J.L., Alberdi, M.T., Azanza, B., Sánchez, B., Frassinetti, D., 2005, The Pleistocene Gomphotheriidae (Proboscidea) from South America: Quaternary International, 126-128, 21-30.

Prado, J.L., Alberdi, M.T., 2008, A cladistic analysis among trilophodont gomphotheres (Mammalia, Proboscidea) with special attention to the South American genera: Palaeontology, 51, 903-915.

Robles-Camacho, J., Corona-Chávez, P., Morales-Gámez, M., Guzmán, A.F., Polaco, O.J., Domínguez-Vázquez, G., Israde-Alcántara, I., Oliveros-Morales, A., 2010, Estratigrafía y paleoambientes asociados a un Gomphotheriidae (Cuvieronius hyodon) en Tzintzunzan, Michoacán, México: Revista Mexicana de Ciencias Geológicas, 27, 530-544.
Rzedowski, J., 1994, Vegetación de México: México, D.F., Limusa, 432 p. Shaw, A.C., 1981, The middle Pleistocene El Golfo local fauna from northwestern Sonora, Mexico: California, U. S. A., California State University, tesis doctoral, $141 \mathrm{p}$.

Shoshani, J., 1996, Para- or monophyly of the gomphotheres and their position within Proboscidea, en Shoshani, J., Tassy, P. (eds.), The Proboscidea: Evolution and Palaeoecology of Elephants and their relatives: Oxford, Oxford University Press, 149-177.

Simpson, G.G., Paula Couto de, C., 1957, The mastodons of Brazil: Bulletin of the American Museum of Natural History, 112,125-190.

Systat Software, Inc., 2006. SigmaPlot for Windows Version 10.0.

Tassy, P., 1977, Le plus ancien squelette de gomphothere (Proboscidea, Mammalia) dans la formation Burdigalienne des sables de l'orléanais France : Memories du Muséum National d' Histoire Naturelle, Ser. C, Sciences de la Terre, 38:1-51.

Tassy, P., 1996, Growth and sexual dimorphism among Miocene elephantoids: the example of Gomphotherium angustidens, en Shoshani, J., Tassy, P. (eds.), The Proboscidea: Evolution and Palaeoecology of Elephants and their relatives: Oxford, Oxford University Press, 92-100.

Torres-Martínez, A, 1981, Faúnula Nanacatla, Pleistoceno del Municipio de Apaxtla, Guerrero (resumen), en V Congreso Nacional de Zoología: México D.F., México, Universidad Nacional Autónoma de México, 121.

Villada, M.M., 1897, Catálogo de la colección de fósiles del Museo nacional: México, Imprenta del Museo Nacional, 79.

Von den Driesch, A., 1976, A guide to the measurement of animal bones from archaeological sites: Peabody Museum Bulletin, 1, 1-137.

Webb, S.D., 1976, Mammalian fauna dynamics of the Great American Interchange: Paleobiology, 2, 220-234.

Manuscrito recibido: Febrero 25, 2013.

Manuscrito corregido recibido: Mayo 5, 2013.

Manuscrito aceptado: Mayo 9, 2013. 